\title{
Properties of solvent-borne acrylic pressure-sensitive adhesives synthesized by a simple approach
}

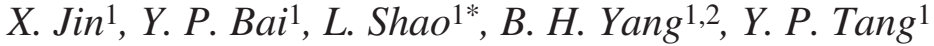 \\ ${ }^{1}$ Department of Polymer Science and Engineering, School of Chemical Engineering and Technology, Harbin Institute of \\ Technology, 150001 Harbin, P.R. China \\ ${ }^{2}$ Northwest Institute of Nuclear Technology, Shanxi Province, 710024 Xi'an, P.R. China
}

Received 2 August 2009; accepted in revised form 30 September 2009

\begin{abstract}
Acrylic polymers are widely used for fabricating pressure-sensitive adhesives (PSAs) with the inherent unique advantages of transparency and superior intrinsic adhesive properties over other polymer-based adhesives. In this study, we have developed and evaluated a method of obtaining by radical copolymerization PSAs for liquid crystalline (LCD) applications. Various factors including the amount of monomers, amount of cross-linker, coating weight, dwell time and thermal treatment are investigated for further optimizing the properties of acrylic polymer based PSAs to meet the emerging strict requirements for practical uses related mainly to holding powder and peel strength. The results illustrate that novel crosslinking reagents coupled with the thermal treatment at $70^{\circ} \mathrm{C}$ can make the resultant PSAs with the improved adhesive properties. The coating weight variation from 10 to $40 \mathrm{~g} / \mathrm{m}^{2}$ can significantly enhance the peel strength from $4.0 \mathrm{~g} / 25 \mathrm{~mm}$ to $12.5 \mathrm{~g} / 25 \mathrm{~mm}$ with about $310 \%$ increment. If the dwell time of PSAs with cross-linking reagent is more than 10 hrs, the peel strength can be reduced down to a suitable value to meet the criterion for use. Therefore, acrylic PSAs with peel strength less than $20 \mathrm{~g} / 25 \mathrm{~mm}$ and holding power above $120 \mathrm{hrs}$ were successfully synthesized by elaborately designing the reaction system, which are practically applicable for advanced industrial applications.
\end{abstract}

Keywords: adhesion, radical polymerization, cross-linking, acrylic polymer, peel strength

\section{Introduction}

With the rapid development of large-format models in liquid crystalline displays (LCD) and the increasing demands on screen-surface functions such as brightness control and shielding ability, various types of optical films have been specially designed and applied, which include the polarizing plate or brightness enhancing film for LCD usage [1]. Before the film assembly on LCD, other processes including punching, transporting and inspecting are necessary. Thus, the application of a protective film is compulsory to protect the surface of the optical films from contaminants or scratching [2]. A sketch of a polarizing plate with a protective film is demonstrated in Figure 1. The sub- strates of the protective film are typically fabricated using polyethylene (PE) or polyethylene terephthalate (PET) with pressure sensitive adhesives (PSAs) coated on one side. PSAs are viscoelastic materials that can adhere to various substrates upon application of light contact pressure in short contact time. The advantages of PSAs include that the

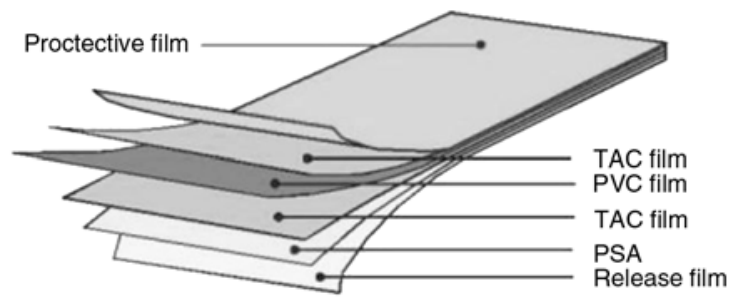

Figure 1. A polarizing plate with a protective film 
resistance of PSAs to debonding is reasonably moderate and the substrates can be delaminated by a suitable force without destroying the targeted laminate components for most of cases. However, for the high value applications such as the application for LCD protective film, the strict requirements for PSAs must be met which include the appropriate peel strength, defect-free, and anti-curvature so that the optical films can cope with the up-to-date technical trends for high quality products.

It is well known that various materials can be used as adhesives [3-5], among which acrylic polymers are the most popular ones for PSA applications because of the inherent unique properties [6]. Acrylic PSAs are transparent, colorless, anti-aging against solar radiation and resistant to oxidation [7]. In addition, PSAs based on acrylic polymers also exhibit intrinsic adhesive properties superior to most of other polymers. Acrylic PSAs, generally borne in an organic solvent, must possess correspondingly suitable $180^{\circ}$ peel strength, high shear strength and appropriate tack [8-10] for optical film applications. The adhesive properties of acrylic PSAs are critically determined by the monomer compositions, the initiators, the crosslinking agents and the tackifiers of acrylic PSAs. To the best of our knowledge, the PSAs used as the surface-protective film for optical film applications have been only reported in some patents. For examples, Tomita [1] has fabricated PSAs comprising the ingredients of: (A) a (meth)acrylic copolymer obtained by copolymerizing 80 to 99 wt $\%$ alkyl (meth)acrylate; (B) an isocyanate crosslinking agent. The synthesized PSAs demonstrated a peel force of $20 \mathrm{gf} / \mathrm{inch}$. Niino et al. [2] have fabricated a protective tape used as optical membranes which demonstrated a low adhesive property. However, no detailed descriptions on PSAs used for the surface-protective film in optical film have been reported in the patents. Considering the importance of this emerging and promising field for PSA applications, a practical study related to the development of the-state-of-the-art materials for such applications should be carried out.
In this study, a simple, practical method of obtaining PSAs for LCD-applications in particular with the films by radical copolymerization of 2-ethylhexyl acrylate (2-EHA), methyl methacrylate (MMA) and acrylic acid (AA) in a typical organic solvent of ethyl acetate (EA) are investigated. The effects of copolymer composition, cross-linking agent content, coating weight and dwell time on adhesive-cohesive characteristics of synthesized PSAs are studied systematically.

\section{Experimental}

\subsection{Materials}

2-EHA, MMA, AA and EA were purchased from Guoyao Chemical Agent Co. Ltd. (Shanghai, China). Benzoylperoxide (BPO) was supplied by Yixing No. 2 Chemical Agent Co. Ltd (Wuxi, China). Cross-linking agents: SC-200 (containing aziridine groups) was obtained from Shanghai Secure Trading Co. Ltd.. All the chemicals were of analytical grade and used without further purification.

\subsection{Preparation of acrylic polymers}

A solution polymerization technique was applied for PSAs synthesis using BPO as a free radical initiator. The PSAs were synthesized with 50 60 parts of 2-EHA, 50 40 parts of MMA, 2 parts of $\mathrm{AA}$, and 100 parts of EA, along with 0.3 part $\mathrm{BPO}$ and the properties and functions of monomers used in this study are listed in Table 1. Monomers (2EHA, MMA, AA) with the predetermined composition were dissolved in EA solvent and placed in a four necked flask equipped with an overhead stirrer, a condenser, a thermometer and an inlet to purge nitrogen gas (for supplying inert reaction environment). Oxygen was eliminated by purging purified nitrogen gas for 20 minutes before initiators were added into the flask. Under the constant temperature maintained at $80^{\circ} \mathrm{C}$, the reaction was continuously carried out for $10 \mathrm{hrs}$ with consistent stirring. The chemical reaction is shown in Figure 2

Table 1. Selected monomers

\begin{tabular}{|c|c|c|c|}
\hline Monomer & $\mathbf{T}_{\mathbf{g}}\left[{ }^{\circ} \mathbf{C}\right]$ & Group & Function \\
\hline 2-EHA & -70 & Tackifying monomer & Provide a certain adhesion \\
\hline MMA & 105 & Hardening monomer & Provide a higher cohesion \\
\hline AA & 106 & Functional groups containing monomer & Provide active cross-linking centers for the later crosss-linking reaction \\
\hline
\end{tabular}




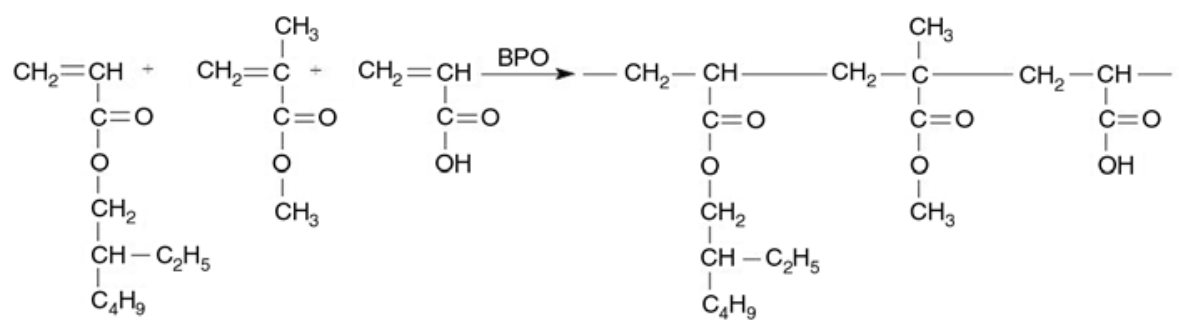

Figure 2. Synthesis reaction of PSA copolymers

Table 2. Component, viscosity and solid content of synthesized PSAs

\begin{tabular}{|c|c|c|c|c|c|}
\hline \multirow{2}{*}{$\begin{array}{c}\text { PSA/ } \\
\text { No. }\end{array}$} & \multicolumn{3}{|c|}{ Monomers (part) } & $\begin{array}{c}\boldsymbol{\eta} \\
{[\mathbf{m P a} \cdot \mathbf{s}]}\end{array}$ & $\begin{array}{c}\text { S.C. } \\
{[\%]}\end{array}$ \\
\hline 1 & 60.0 & 40.0 & 2 & 3832 & 48.1 \\
\hline 2 & 57.5 & 42.5 & 2 & 4108 & 50.3 \\
\hline 3 & 55.0 & 45.0 & 2 & 3910 & 46.7 \\
\hline 4 & 52.5 & 47.5 & 2 & 4214 & 53.3 \\
\hline 5 & 50.0 & 50.0 & 2 & 4048 & 47.9 \\
\hline
\end{tabular}

and the properties of synthesized PSAs according to different compositions were shown in Table 2.

\subsection{Preparation of PSA tapes}

Cross-linking reagents were diluted to $10 \mathrm{wt} \%$ concentration in EA which was added into the acrylic copolymer solution (about 50\% solid content) with high speed stirring for mixing about 20 minutes. The resultant solution was further diluted to a desired concentration (about $30 \%$ by weight) to meet the requirement of coating on a PET film. After being dried at $120^{\circ} \mathrm{C}$ for 3 minutes, PSA tapes with coating weight of $30 \mathrm{~g} / \mathrm{m}^{2}$ were ready for characterizations. And the purpose of the 3 minutes drying process is to eliminate the solvent within the PSA and to initiate the cross-linking reaction, in general, to form the product PSA tape.

\section{4. $180^{\circ}$ Peel strength test}

Strips of PSA tape samples $(25 \mathrm{~mm} \times 250 \mathrm{~mm})$ were attached to the surfaces of stainless-steel test panel by rolling twice with a $2 \mathrm{~kg}$ rubber roller at a speed of $10 \mathrm{~mm}$ per second. The pressed PSA tapes were kept for $20 \mathrm{~min}$ at $23^{\circ} \mathrm{C}$ with $50 \%$ relative humidity. $180^{\circ}$ peel strength was measured with a peeling speed of $300 \mathrm{~mm} / \mathrm{min}$ by using an Adhesion/ Release Tester AR-1000 (ChemInstrument, USA). The average peel force measured during the entire peeling process was recorded.

\subsection{Holding power test}

A square of PSA coated tape, $25 \mathrm{~mm} \times 25 \mathrm{~mm}$, was tightly contact with the surface of the testing plate. Roll twice in each direction with a $2 \mathrm{~kg}$ rubber roller at a speed of $10 \mathrm{~mm}$ per second. Place the testing plate into the rack of jig and attach the $1 \mathrm{~kg}$ weight to the free end of the testing strip after storing for 20 minutes at $23^{\circ} \mathrm{C}$ with $50 \%$ relative humidity. Holding power was expressed as the time taken for the strips to shear from the test plate.

\section{Results and discussion}

\subsection{Influence of MMA content on properties of PSAs}

The main reason that monomer of MMA is used to modify the cohesive strength of PSAs is due to the inherent high $T_{g}$ of MMA monomers as Table 1 shown [11]. After the comprehensive investigations on the composition during the radical polymerization, the effect of MMA content on the peel strength is illustrated in Figure 3. When the MMA content increases from 40 to $50 \%$, the holding power of PSAs correspondingly increases from around 8 to $36 \mathrm{hrs}$ and the peel strength decreases from $90 \mathrm{~g} / 25 \mathrm{~mm}$ to $10 \mathrm{~g} / 25 \mathrm{~mm}$. Therefore, the increment in the MMA content can significantly

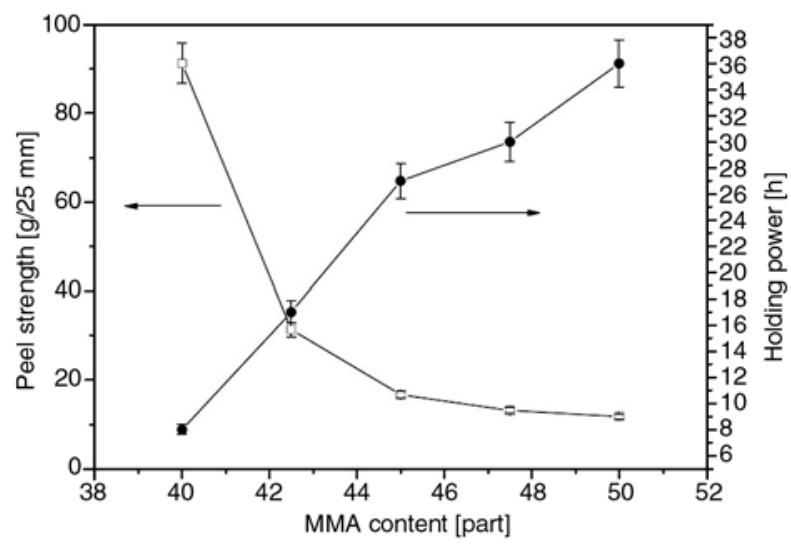

Figure 3. Influence of MMA content on peel strength and holding power 
enhance the holding power of PSAs and decrease the peel strength of PSAs. Typically, MMA provides the cohesion properties and 2-EHA contributes to adhesion properties of PSAs. The content of 2-EHA decreases with the MMA content which results in the enhanced holding power and the weaken peel strength. However, when the MMA content exceeds 45 part (to 50 part), the decrement of peel strength is not significantly obvious (from around $18 \mathrm{~g} / 25 \mathrm{~mm}$ to $10 \mathrm{~g} / 25 \mathrm{~mm}$ ). Since the purpose of this study is to prepare PSAs with the peel strength less than $20 \mathrm{~g} / 25 \mathrm{~mm}$ which can meet the industrial requirements, PSAs with 45 part MMA is enough for peel strength requirements and selected for the following study.

\subsection{Influence of cross-linking reagents on properties of PSAs}

Various chemical compounds [1, 12, 13] including multifunctional isocyanates, multifunctional ethyleneimines and epoxy resins, can be utilized to cross-link PSAs for the manipulation of resultant PSA properties. In this study, cross-linking reagents of SC-200 are specially utilized to achieve high holding power and good heat resistant property. The effects of SC-200 content on the properties of PSAs with or without thermal treatment are demonstrated in Figure 4. The holding power for all PSAs (with or without thermal treatment) containing cross-linking reagents above 0.25 part was enhanced up to above $120 \mathrm{~h}$. For the samples without thermal treatment, the increase of cross-linking reagent content from 0.25 to 1.0 part can decrease

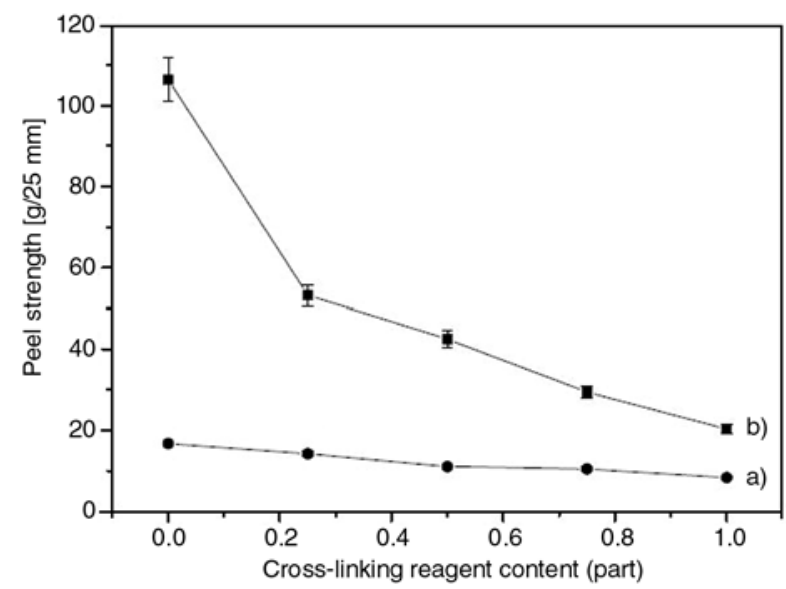

Figure 4. Influence of the amount of cross-linker on peel strength of (a) without high temperature treatment and (b) treated at $70^{\circ} \mathrm{C}$ for $24 \mathrm{~h}$

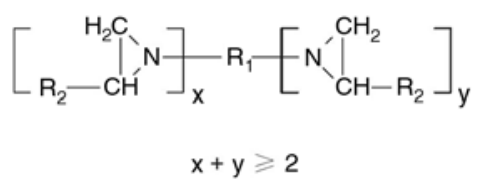

Figure 5. Structure of Cross-linker SC-200

the peel strength from $16.7 \mathrm{~g} / 25 \mathrm{~mm}$ to $8.3 \mathrm{~g} /$ $25 \mathrm{~mm}$. To illustrate the reaction mechanism, the chemical structure of SC-200 is shown in Figure 5, in which $R_{1}$ is an $n$-valent organic or inorganic group and $R_{2}$ is $-\mathrm{H}$ or $-\mathrm{CH}_{3}$. The cross-linking reaction between original PSAs and SC-200 is mainly based on the carboxyl groups offered by the AA functional component within PSA copolymers and the aziridine groups provided by SC-200. During the cross-linking reaction, the carboxyl groups act as proton donors supplying protons to attack the nitrogen atoms for protonation and cause the opening of the enimine rings. Then, a carbocation is generated as an intermediate which is free of the counterion $\mathrm{COO}^{-}$. Based on the above analysis, the sketch of the ring-opening reaction is illustrated in Figure 6, while the mechanism of the entire crosslinking reaction is demonstrated in Figure 7.

As previously mentioned, cross-linking process has been considered as an important factor for the final properties of PSAs for practical applications [14, 15]. The cross-linking reactions drastically inhibit the mobility of polymer molecules by forming chemical bonds among the chains of PSAs and correspondingly increase the molecular weight of polymers. After that, the cross-linked PSAs can not melt any more because of the cross-linked network formed. However, the cross-linked PSAs can become soft at a high temperature. Besides, the cross-linked PSAs undergo decomposition above certain temperatures. Owing to the formation of chemical bonds, an increase in cohesion can be obtained during the curing of the PSA coating layer

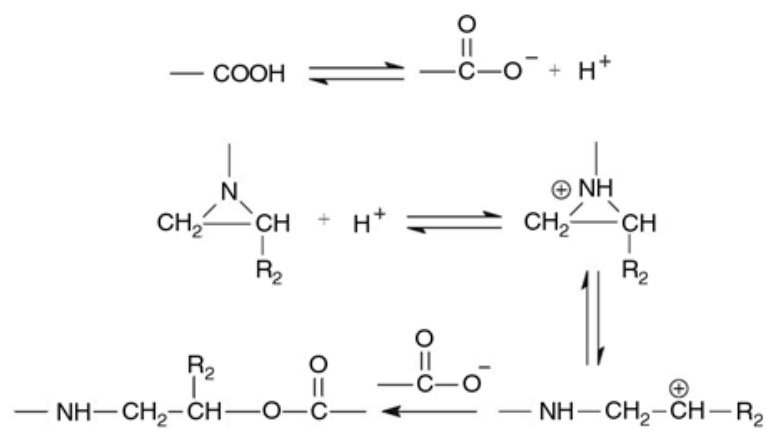

Figure 6. Sketch of the ring-opening reaction 


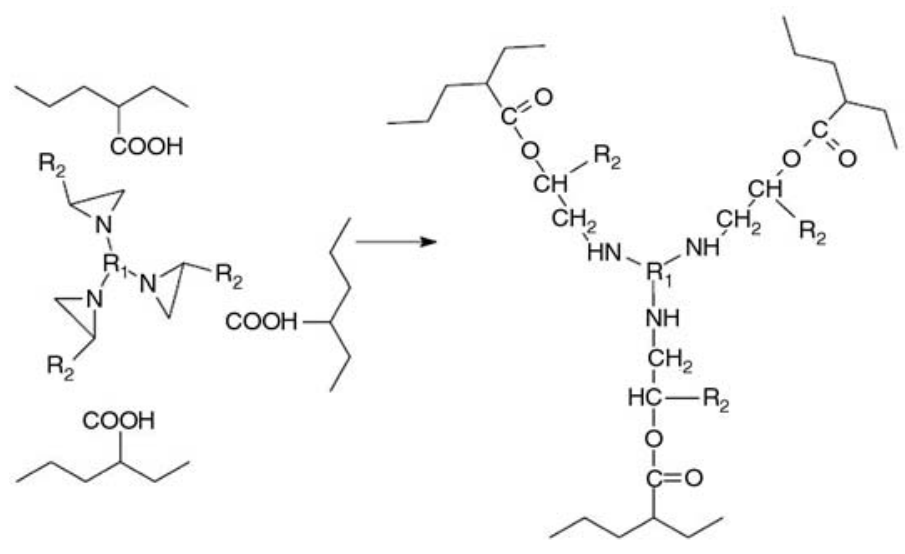

Figure 7. Cross-linking of PSA initiated by SC-200

in the drying step, in which the cross-linking reaction occurs [16, 17].

Figure 4 also illustrates the influences of crosslinking reagents on the heat resistance of PSA. After the PSA strips adhered completely on the stainless-steel plates, the specimens were transferred to an oven maintained at $70^{\circ} \mathrm{C}$. The high temperature treatment persisted for $24 \mathrm{~h}$. After the specimens cooled to room temperature, peel strength testing was performed to characterize the heat resistant performance of PSAs. Interestingly, after high temperature treatment, the peel strength of PSAs increases significantly. For example, the peel strength of PSA without cross-linking reagent increases about 6 times after $70^{\circ} \mathrm{C}$ thermal treatment. In addition, cohesive failure shows up during the peel strength test. Gratefully, with the addition of cross-linker, the increment of peel strength decreased which is much suitable for PSA applications.

\subsection{Influence of coating weight on peel strength}

The following investigations are conducted in order to study the influence of the coating weight of PSAs on peel strength. The PSA containing 45 parts MMA is mixed with 0.5 part cross-linker, and then is coated on a piece of PET film. The increase of coating weight from 10 to $40 \mathrm{~g} / \mathrm{m}^{2}$ enhances the peel strength from $4.0 \mathrm{~g} / 25 \mathrm{~mm}$ to $12.5 \mathrm{~g} / 25 \mathrm{~mm}$ which indicates the significant increment of peel strength about $310 \%$ (as Figure 8 shown), while the holding power property of each specimen is still up to $120 \mathrm{hrs}$. The isolation of a PSA tape from a substrate is concomitant with a

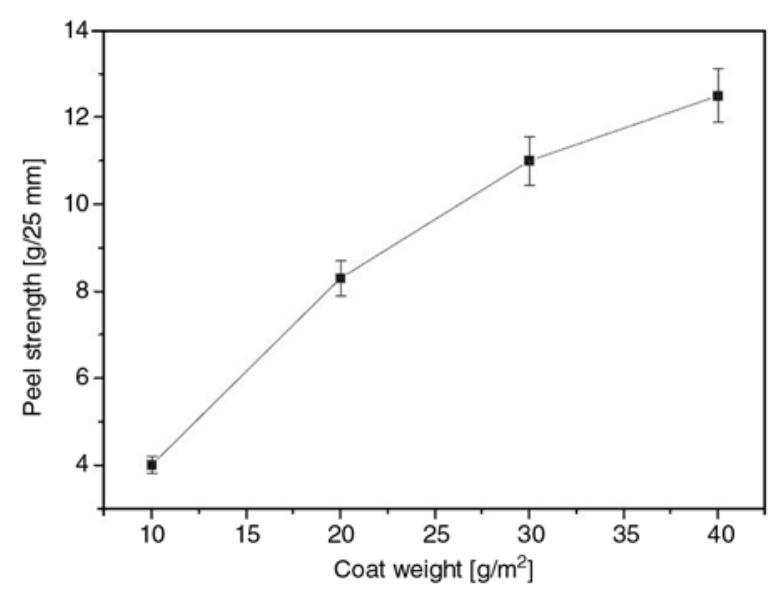

Figure 8. Influence of coating weight on peel strength

certain number of non-equilibrium and dissipative processes which include large bulk deformations [18]. In some cases, these deformations involve cavitations and fibrillations, which both significantly contribute to the energy of separation [19]. The increase of the coating weight has a noticeable effect on intensifying the deformations, thus more dissipative the PSA tape exhibits. As a result, the larger peel strength is achieved with the increase of coating weight.

\subsection{Dwell time effect on properties of PSAs}

Dwell effect of PSAs with cross-linking reagent on peel strength is characterized further for practical applications. The peel strength of nascent PSAs declined gradually with the dwell time. The decreasing trend is not so obvious after $10 \mathrm{hrs}$ since the PSA tapes are prepared (as Figure 9 shown). This phenomenon indicates that an acceptable cross-linking degree in PSAs can be achieved after 10 hrs dwell of nascent PSAs. 


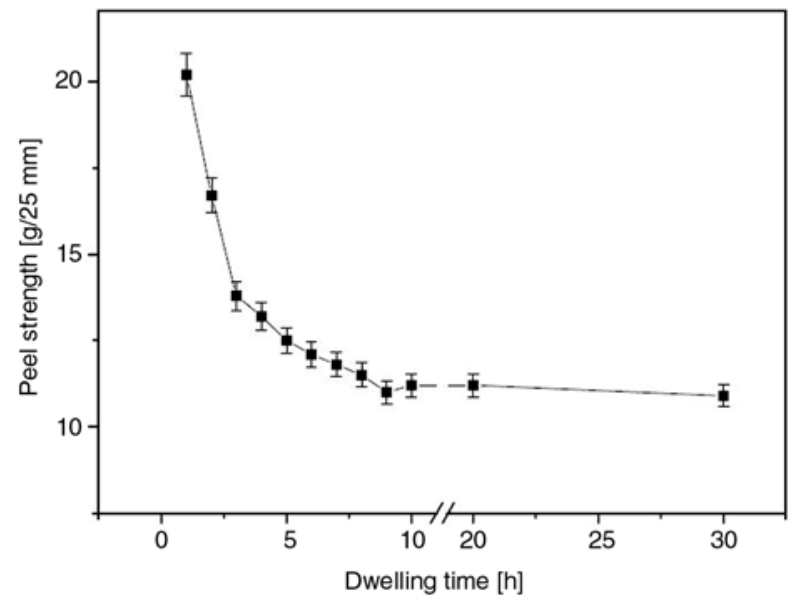

Figure 9. The influence of dwell time on peel strength

\section{Conclusions}

In summary, PSAs with properties of peel strength no more than $20 \mathrm{~g} / 25 \mathrm{~mm}$ and holding power above $120 \mathrm{hrs}$ are successfully synthesized with a simple radical polymerization method. PSA tapes possessing targeted properties can be fabricated to meet the special requirements for industrial usages. The increase of MMA content decreases the peel strength and improves the holding power. The incorporation of cross-linking reagents of SC-200 is crucial for improving the adhesive properties of acrylic PSAs. The increase of cross-linking reagents decreases the peel strength, improves the holding power ( $>120 \mathrm{hrs})$, and enhances the heat resistance. Besides, the increase of coating weight enhances the peel strength of PSAs. Extending the dwelling time of PSA tapes containing cross-linking reagent has a positive effect on decreasing the peel strength.

\section{Acknowledgements}

Dr. Shao thanks for the support from the Specialized Research Fund for the Doctoral Program of Higher Education (20070213004), Harbin Science and Technology Innovation Talent Funds (contract grant number: 2006RFLXG015) and the Scientific Research Foundation for the Returned Oversea Chinese Scholars, State Education Ministry.

\section{References}

[1] Tomita K.: Pressure-sensitive adhesive for surfaceprotective film and surface-protective film. WO/2005/ 000989 (2005).
[2] Niino T., Imono S., Okumura K., Sano K.: Protective tape used for optical membrane. US Patent 7270877 B2, USA (2007).

[3] Wei S-Q., Bai Y-P., Shao L.: A novel approach to graft acrylates onto commercial silicones for release film fabrications by two-step emulsion synthesis. European Polymer Journal, 44, 2728-2736 (2008). DOI: $\underline{10.1016 / j . e u r p o l y m j .2008 .04 .025}$

[4] Patel H. J., Patel M. G., Patel A. K., Patel K. H., Patel R. M.: Synthesis, characterization and antimicrobial activity of important heterocyclic acrylic copolymers. Express Polymer Letters, 2, 727-734 (2008). DOI: $10.3144 /$ expresspolymlett.2008.86

[5] Jeusette M., Peeterbroeck S., Simal F., Cossement D., Roose P., Leclere Ph., Dubois Ph., Hecq M., Lazzaroni R.: Microscopic morphology of blends between a new 'all-acrylate' radial block copolymer and a rosin ester resin for pressure sensitive adhesives. European Polymer Journal, 44, 3931-3940 (2008).

DOI: $\underline{10.1016 / j . e u r p o l y m j .2008 .09 .003}$

[6] Czech Z., Butwin A., Herko E., Hefczyc B., Zawadiak J.: Novel azo-peresters radical initiators used for the synthesis of acrylic pressure-sensitive adhesives. Express Polymer Letters, 2, 277-283 (2008). DOI: $10.3144 /$ expresspolymlett.2008.33

[7] Taghizadeh S. M., Mirzadeh H., Barikani M., Yousefi M.: Miscibility and tack of blends of poly(vinylpyrrolidone)/acrylic pressure-sensitive adhesive. International Journal of Adhesion and Adhesives, 29, 302 308 (2009).

DOI: 10.1016/j.ijadhadh.2008.06.006

[8] Xu G. Z. H., Dong J. P., Zhang J. G., Severtson S. J., Houtman C. J., Gwin L. E.: Characterizing the distribution of nonylphenol ethoxylate surfactants in waterbased pressure-sensitive adhesive films using atomic-force confocal Raman microscopy. The Journal of Physical Chemistry B, 112, 11907-11914 (2008).

[9] Taghizadeh S. M., Mirzadeh H., Barikani M., Yousefi M.: The effect of poly(vinylpyrrolidone) concentration on peel strength of acrylic/PVP pressure sensitive adhesive blends. Iranian Polymer Journal, 16, 279285 (2007).

[10] Bai Y-P., Zhao L., Shao L.: Hybrid emulsifiers enhancing polymerization stabilities and properties of pressure sensitive adhesives. Journal of Applied Polymer Science, 115, 1125-1130 (2009). DOI: $10.1002 / a p p .31211$

[11] Yang Y. K., Lv F. T.: Technical handbook of PSA products. Chemical Industry Press, Beijing (2004).

[12] Gardeski T. F.: A high performance epoxy based coverlay and bond ply adhesive with heat activated cure mechanism. European Patent 0436745A1, EU (1989).

[13] Saiki N., Yamazaki O., Ebe K.: UV/heat dual-durable adhesive tapes for fabricating stacked packages of semiconductors. Journal of Applied Polymer Science, 108, 1178-1183 (2008). DOI: $\underline{10.1002 / a p p .27663}$ 
[14] Yamamoto M., Nakano F., Doi T., Moroishi Y.: Synthesis and PSA performance study for novel acrylic and butyl acrylate block copolymers. International Journal of Adhesion and Adhesives, 22, 37-40 (2002). DOI: 10.1016/S0143-7496(01)00034-3

[15] Moon J. H., Shul Y. G., Hong S. Y., Choi Y. S., Kim H. T.: A study on UV-curable adhesives for optical pick-up: I. Photo-initiator effects. International Journal of Adhesion and Adhesives, 25, 301-312 (2005). DOI: $10.1016 /$ j.ijadhadh.2004.09.003

[16] Verdier C., Piau J-M., Benyahia L.: Peeling of acrylic pressure sensitive adhesives: Cross-linked versus uncross-linked adhesives. Journal of Adhesion, 68, 93-116 (1998).

DOI: $\underline{10.1080 / 00218469808029581}$
[17] Brostow W.: Time-stress correspondence in viscoelastic materials: An equation for the stress and temperature shift factor. Material Research Innovations, 3, 218-225 (2000).

DOI: $10.1007 / \mathrm{s} 100190000054$

[18] Wang T., Lei C-H., Dalton A. B., Creton C., Lin Y., Fernando K. A. S., Sun Y-P., Manea M., Asua J. M., Keddie J. L.: Waterborne, nanocomposite pressuresensitive adhesives with high tack energy, optical transparency, and electrical conductivity. Advanced Materials, 18, 2730-2734 (2006). DOI: $10.1002 / \mathrm{adma} .200601335$

[19] Zosel A.: The effect of fibrillation on the tack of pressure sensitive adhesives. International Journal of Adhesion and Adhesives, 18, 265-271 (1998). DOI: $\underline{10.1016 / \mathrm{S} 0143-7496(98) 80060-2}$ 